\title{
On a tensor-analogue of the Schur product
}

\author{
K. Sumesh and V.S. Sunder \\ Institute of Mathematical Sciences \\ Chennai 600113 \\ INDIA \\ email: sumeshkpl@gmail.com, sunder@imsc.res.in
}

July 16, 2018

\begin{abstract}
We consider the tensorial Schur product $R \circ^{\otimes} S=\left[r_{i j} \otimes s_{i j}\right]$ for $R \in M_{n}(\mathcal{A}), S \in M_{n}(\mathcal{B})$, with $\mathcal{A}, \mathcal{B}$ unital $C^{*}$-algebras, verify that such a 'tensorial Schur product' of positive operators is again positive, and then use this fact to prove (an apparently marginally more general version of) the classical result of Choi that a linear map $\phi: M_{n} \rightarrow M_{d}$ is completely positive if and only if $\left[\phi\left(E_{i j}\right)\right] \in M_{n}\left(M_{d}\right)^{+}$, where of course $\left\{E_{i j}: 1 \leq i, j \leq n\right\}$ denotes the usual system of matrix units in $M_{n}\left(:=M_{n}(\mathbb{C})\right)$. We also discuss some other corollaries of the main result.
\end{abstract}

\section{The result}

We start with some notation: (We assume, for convenience, that all our $C^{*}$ algebras are unital.) We denote an element of a matrix algebra by capital letters, such as $R$, and denote its entries by either $[R]_{i j}$ or the corresponding lower case letter $r_{i j}$. This is primarily because $\left[R^{*}\right]_{i j}=\left(r_{j i}\right)^{*} \neq\left[R^{*}\right]_{j i}$ !

Definition 1.1. 1. If $\mathcal{A}, \mathcal{B}$ are $C^{*}$-algebras, and $\phi: M_{n} \rightarrow \mathcal{B}$ is a positive map, define $\phi_{\mathcal{A}}: A \otimes M_{n} \rightarrow \mathcal{A} \otimes_{\text {alg }} \mathcal{B}$ (where $\mathcal{A} \otimes_{\text {alg }} \mathcal{B}$ denotes the algebraic tensor product of $\mathcal{A}$ and $\mathcal{B}$ ) by $\phi_{\mathcal{A}}=i d_{\mathcal{A}} \otimes \phi$. 
2. If $A=\left[a_{i j}\right] \in M_{n}(\mathcal{A}), B=\left[b_{i j}\right] \in M_{n}(\mathcal{B})$, define $A \circ^{\otimes} B=\left[a_{i j} \otimes b_{i j}\right] \in$ $M_{n}\left(\mathcal{A} \otimes_{\text {alg }} \mathcal{B}\right)$.

For later use, we isolate a lemma, whose elementary verification we omit.

Lemma 1.2. The map $\pi: M_{n}(\mathcal{A}) \otimes M_{k} \rightarrow M_{n k}(\mathcal{A})$ defined by

$$
[\pi(R \otimes C)]_{i \alpha, j \beta}=c_{\alpha \beta} r_{i j}
$$

is a $C^{*}$-algebra isomorphism for any $C^{*}$-algebra $\mathcal{A}$; in the sequel, we shall simply use this $\pi$ to make the identification $M_{n}(\mathcal{A}) \otimes M_{k}=M_{n k}(\mathcal{A})$. In particular, $\mathcal{A} \otimes M_{k}=M_{k}(\mathcal{A})$.

REMARK 1.3. There is clearly a right version of the above Lemma: i.e., $M_{k} \otimes M_{n}(\mathcal{A})=M_{k n}(\mathcal{A})$.

Proposition 1.4.

$$
R \in M_{n}(\mathcal{A})^{+}, S \in M_{n}(\mathcal{B})^{+} \Rightarrow R \circ^{\otimes} S \in M_{n}(\mathcal{C})^{+},
$$

where $\mathcal{C}$ denotes - here and in the rest of this short note - any $C^{*}$-algebra containing $\mathcal{A} \otimes_{\text {alg }} \mathcal{B}$. In particular,

$$
\sum_{i, j=1}^{n} r_{i j} \otimes s_{i j} \in M_{n}(\mathcal{C})^{+} .
$$

Proof. To deduce eqn. (1.3) from eqn. (1.2), we let $1_{n} \in M_{n \times 1}(\mathcal{C})^{+}$be the $n \times 1$ column-vector with all entries equal to $1_{\mathcal{A}} \otimes 1_{\mathcal{B}}$, and note that

$$
\sum_{i, j=1}^{n} r_{i j} \otimes s_{i j}=1_{n}^{*}\left(R \circ^{\otimes} S\right) 1_{n} .
$$

Now for the slightly less immediate eqn. (1.2). By assumption, $R \otimes S \in$ $M_{n^{2}}(\mathcal{C})^{+}$.

In the sequel all the variables $i, j, k, l, p, q$ will range over the set $\{1,2, \ldots, n\}$ and we shall simply write $\sum_{k}$ for $\sum_{k=1}^{n}$.

Now define $V \in M_{n \times n^{2}}(\mathcal{C})$ by

$$
[V]_{i, p q}=\delta_{p i} \delta_{q i}\left(1_{A} \otimes 1_{B}\right) .
$$


Then,

$$
\begin{aligned}
{\left[V(R \otimes S) V^{*}\right]_{i j} } & =\sum_{p, q, k, l}[V]_{i, p q}[R \otimes S]_{p q, k l}\left[V^{*}\right]_{k l, j} \\
& =[R \otimes S]_{i i, j j} \\
& =r_{i j} \otimes s_{i j}
\end{aligned}
$$

and so,

$$
V(R \otimes S) V^{*}=R \circ^{\otimes} S .
$$

The proof of the Proposition is complete.

REMARK 1.5. Note that the proof shows that $R \circ^{\otimes} S \in M_{n}\left(\mathcal{A} \otimes_{\text {alg }} \mathcal{B}\right)$.

The classical result of Choi alluded to in the abstract is the equivalence 2. $\Leftrightarrow 3$. in the following Corollary, for the case $\mathcal{B}=M_{d}$ (see [1]).

COROLlary 1.6. The following conditions on a linear map $\phi: M_{n} \rightarrow \mathcal{B}$ are equivalent:

1. For any $C^{*}$-algebra $\mathcal{A}$, the map $\phi_{\mathcal{A}}\left(:=i d_{\mathcal{A}} \otimes \phi\right): \mathcal{A} \otimes M_{n} \rightarrow \mathcal{C}$ is a positive map for any $C^{*}$-algebra $\mathcal{C}$ as in Proposition 1.4 .

2. The map $\phi$ is $C P 1$

3. $\left[\phi\left(E_{i j}\right)\right] \in M_{n}(\mathcal{B})^{+}$.

Proof. We only prove the non-trivial implication 3. $\Rightarrow 1$. if $R \in\left(\mathcal{A} \otimes M_{n}\right)^{+}=$ $M_{n}(\mathcal{A})^{+}$, and if $R=\left[r_{i j}\right]$, then

$$
\begin{aligned}
\phi_{\mathcal{A}}(R) & =\left(i d_{\mathcal{A}} \otimes \phi\right)\left(\sum_{i j} r_{i j} \otimes E_{i j}\right) \\
& =\sum_{i j} r_{i j} \otimes \phi\left(E_{i j}\right) \\
& \in M_{n}(\mathcal{C})^{+}
\end{aligned}
$$

by eqn. (1.3).

\footnotetext{
${ }^{1}$ For an explanation of terms like $\mathrm{CP}$ (= completely positive) and operator system, the reader may consult [3], for instance.
} 
Corollary 1.7. Let $R \in M_{n}(\mathcal{A})^{+}$. Then the map $M_{n}(\mathcal{B}) \ni S \stackrel{L_{R}}{\rightarrow} R \circ^{\otimes} S \in$ $M_{n}(\mathcal{C})$ is CP. In particular $R \in M_{n}^{+} \Rightarrow M_{n} \ni S \rightarrow R \circ S \in M_{n}$ is also CP.

Proof. To avoid confusion, we use Greek letters $\alpha, \beta$ etc., to denote elements of $\{1,2, \cdots, k\}$ and English letters $i, j$ etc. to denote elements of $\{1,2, \cdots, n\}$. Suppose $[\hat{S}] \in M_{k n}(\mathcal{B})^{+}=M_{k}\left(M_{n}(\mathcal{B})\right)^{+}$is given by $[\hat{S}]_{\alpha i, \beta j}:=$ $\left[S_{\alpha, \beta}\right]_{i, j}$ (see Lemma 1.2), where of course $\left.S_{\alpha \beta} \in M_{n}(\mathcal{B}) \forall \alpha, \beta\right)$. Let $J_{k} \in M_{k}$ be (the all 1 matrix) given by $\left[J_{k}\right]_{\alpha \beta}=1 \forall \alpha, \beta$. Then we see that $J_{k} \geq 0$ (in fact $J_{k} / k$ is a projection) and so,

$$
\begin{aligned}
{\left[L_{R}\left(S_{\alpha \beta}\right)\right] } & =\left[\left[r_{i j} \otimes\left[S_{\alpha \beta}\right]_{i j}\right]\right] \\
& =\left[\left[\left[J_{k}\right]_{\alpha \beta} r_{i j} \otimes\left[S_{\alpha \beta}\right]_{i j}\right]\right] \\
& \left.=\left[\left[J_{k} \otimes R\right]_{\alpha i, \beta j} \otimes[\hat{S}]_{\alpha i, \beta j}\right] \quad \text { (see Remark 1.3. }\right) \\
& =\left(J_{k} \otimes R\right) \circ^{\otimes} \hat{S} \\
& \geq 0,
\end{aligned}
$$

by Proposition 1.4 applied with $R, n, S$ there replaced by $J_{k} \otimes R, k n, \hat{S}$, since $\left[J_{k} \otimes R\right]_{\alpha i, \beta j}=r_{i j} \forall \alpha, \beta$. The second statement of the Corollary is just the specialisation of the first statement to $\mathcal{A}=\mathcal{B}=\mathbb{C}$.

REMARK 1.8. The special case $n=1$ of Corollary 1.7 perhaps merits singling out: If $r \in \mathcal{A}^{+}$, then the map $\mathcal{B} \ni s \stackrel{L_{\rightarrow}}{\rightarrow} r \otimes s \in \mathcal{C}$ is $C P$.

REMARK 1.9. It should be clear that there is a 'right' version of all the 'left' statements discussed above.

The proofs suggest that these results might well admit formulations in the language of operator systems; however, we suspect that such 'generalisations' will follow from nuclearity of $M_{n}$ and the flexibility in the choice of $\mathcal{C}$ in our formulation, in view of the Choi-Effros theorem (see [2]).

\section{References}

[1] M. D. Choi, Completely positive linear maps on complex matrices, Linear Algebra and Appl. 10 (1975), 285-290.

[2] M. D. Choi and E. G. Effros, Injectivity and operator spaces, J. of Functional Analysis 24 (1977), no. 2, 156-209. 
[3] G. Pisier, Introduction to Operator Space Theory. LMS Lecture Note Series 294, Cambridge University Press, 2003. 